Supplement of Geosci. Model Dev. Discuss., 8, 4823-4849, 2015

http://www.geosci-model-dev-discuss.net/8/4823/2015/

doi:10.5194/gmdd-8-4823-2015-supplement

(c) Author(s) 2015. CC Attribution 3.0 License.

(c) (i)

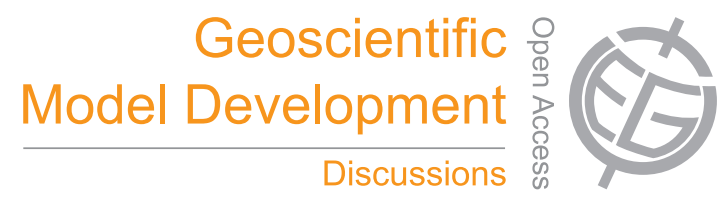

Supplement of

\title{
Development of a chlorine chemistry module for the Master Chemical Mechanism
}

\section{K. Xue et al.}

Correspondence to: L. K. Xue (xuelikun@sdu.edu.cn)

The copyright of individual parts of the supplement might differ from the CC-BY 3.0 licence. 
Table S1. Summary of organic reactions added to the MCM to represent the Cl chemistry ${ }^{\text {a }}$

\begin{tabular}{|c|c|c|}
\hline Reactions & $k\left(\mathrm{~cm}^{3}\right.$ molecules $\left.^{-1} \mathrm{~s}^{-1}\right)$ & Source \\
\hline \multicolumn{3}{|c|}{ Aldehydes $+\mathrm{Cl}$} \\
\hline $\mathrm{HCHO}+\mathrm{Cl} \rightarrow \mathrm{HCl}+\mathrm{HO} 2+\mathrm{CO}$ & $8.1 \times 10^{-11} \times \exp (-34 / \mathrm{T})$ & IUPAC \\
\hline $\mathrm{CH} 3 \mathrm{CHO}+\mathrm{Cl} \rightarrow \mathrm{CH} 3 \mathrm{CO} 3+\mathrm{HCl}$ & $7.92 \times 10^{-11}$ & IUPAC \\
\hline $\mathrm{CH} 3 \mathrm{CHO}+\mathrm{Cl} \rightarrow \mathrm{HCOCH} 2 \mathrm{O} 2+\mathrm{HCl}$ & $8.0 \times 10^{-13}$ & IUPAC \\
\hline $\mathrm{C} 2 \mathrm{H} 5 \mathrm{CHO}+\mathrm{Cl} \rightarrow \mathrm{C} 2 \mathrm{H} 5 \mathrm{CO} 3+\mathrm{HCl}$ & $1.3 \times 10^{-10}$ & IUPAC \\
\hline $\mathrm{C} 3 \mathrm{H} 7 \mathrm{CHO}+\mathrm{Cl} \rightarrow \mathrm{BUTALO} 2+\mathrm{HCl}$ & $5.5 \times 10^{-12} \times \exp (410 / \mathrm{T})$ & $\mathrm{b}$ \\
\hline $\mathrm{C} 3 \mathrm{H} 7 \mathrm{CHO}+\mathrm{Cl} \rightarrow \mathrm{C} 3 \mathrm{H} 7 \mathrm{CO} 3+\mathrm{HCl}$ & $3.1 \times 10^{-11} \times \exp (410 / \mathrm{T})$ & $\mathrm{b}$ \\
\hline $\mathrm{IPRCHO}+\mathrm{Cl} \rightarrow$ IBUTALBO2 $+\mathrm{HCl}$ & $2.2 \times 10^{-12} \times \exp (410 / \mathrm{T})$ & $\mathrm{b}$ \\
\hline $\mathrm{IPRCHO}+\mathrm{Cl} \rightarrow \mathrm{IBUTALCO} 2+\mathrm{HCl}$ & $2.4 \times 10^{-12} \times \exp (410 / \mathrm{T})$ & $\mathrm{b}$ \\
\hline $\mathrm{IPRCHO}+\mathrm{Cl} \rightarrow \mathrm{IPRCO} 3+\mathrm{HCl}$ & $3.7 \times 10^{-11} \times \exp (410 / \mathrm{T})$ & $\mathrm{b}$ \\
\hline $\mathrm{C} 4 \mathrm{H} 9 \mathrm{CHO}+\mathrm{Cl} \rightarrow \mathrm{C} 4 \mathrm{CHOBO} 2+\mathrm{HCl}$ & $7.3 \times 10^{-12} \times \exp (448 / \mathrm{T})$ & $\mathrm{b}$ \\
\hline $\mathrm{C} 4 \mathrm{H} 9 \mathrm{CHO}+\mathrm{Cl} \rightarrow \mathrm{C} 4 \mathrm{H} 9 \mathrm{CO} 3+\mathrm{HCl}$ & $3.1 \times 10^{-11} \times \exp (448 / \mathrm{T})$ & $\mathrm{b}$ \\
\hline $\mathrm{BENZAL}+\mathrm{Cl} \rightarrow \mathrm{C} 6 \mathrm{H} 5 \mathrm{CO} 3+\mathrm{HCl}$ & $3.6 \times 10^{-11} \times \exp (225 / \mathrm{T})$ & $\mathrm{b}$ \\
\hline \multicolumn{3}{|c|}{ Ketones $+\mathrm{Cl}$} \\
\hline $\mathrm{CH}_{3} \mathrm{COCH}_{3}+\mathrm{Cl} \rightarrow \mathrm{CH}_{3} \mathrm{COCH}_{2} \mathrm{O}_{2}+\mathrm{HCl}$ & $1.5 \times 10^{-11} \times \exp (-590 / \mathrm{T})$ & IUPAC \\
\hline $\mathrm{MEK}+\mathrm{Cl} \rightarrow \mathrm{MEKAO}_{2}+\mathrm{HCl}$ & $1.4 \times 10^{-11} \times \exp (80 / \mathrm{T})$ & IUPAC \\
\hline $\mathrm{MEK}+\mathrm{Cl} \rightarrow \mathrm{MEKBO}_{2}+\mathrm{HCl}$ & $1.4 \times 10^{-11} \times \exp (80 / \mathrm{T})$ & IUPAC \\
\hline $\mathrm{MEK}+\mathrm{Cl} \rightarrow \mathrm{MEKCO} 2+\mathrm{HCl}$ & $2.4 \times 10^{-12} \times \exp (80 / \mathrm{T})$ & IUPAC \\
\hline $\mathrm{MPRK}+\mathrm{Cl} \rightarrow \mathrm{CO} 2 \mathrm{C} 54 \mathrm{O} 2+\mathrm{HCl}$ & $9.6 \times 10^{-11}$ & $\mathrm{~b}$ \\
\hline $\mathrm{MPRK}+\mathrm{Cl} \rightarrow \mathrm{MPRKAO} 2+\mathrm{HCl}$ & $2.1 \times 10^{-11}$ & $\mathrm{~b}$ \\
\hline $\mathrm{DIEK}+\mathrm{Cl} \rightarrow \mathrm{DIEKAO} 2+\mathrm{HCl}$ & $2.4 \times 10^{-11}$ & $\mathrm{~b}$ \\
\hline $\mathrm{DIEK}+\mathrm{Cl} \rightarrow \mathrm{DIEKBO} 2+\mathrm{HCl}$ & $2.4 \times 10^{-11}$ & $\mathrm{~b}$ \\
\hline $\mathrm{MIPK}+\mathrm{Cl} \rightarrow \mathrm{MIPKAO} 2+\mathrm{HCl}$ & $3.5 \times 10^{-11}$ & $\mathrm{~b}$ \\
\hline $\mathrm{MIPK}+\mathrm{Cl} \rightarrow \mathrm{MIPKBO} 2+\mathrm{HCl}$ & $3.2 \times 10^{-11}$ & $\mathrm{~b}$ \\
\hline $\mathrm{HEX} 2 \mathrm{ONE}+\mathrm{Cl} \rightarrow \mathrm{HEX} 2 \mathrm{ONAO} 2+\mathrm{HCl}$ & $1.56 \times 10^{-10}$ & $\mathrm{~b}$ \\
\hline $\mathrm{HEX} 2 \mathrm{ONE}+\mathrm{Cl} \rightarrow \mathrm{HEX} 2 \mathrm{ONBO} 2+\mathrm{HCl}$ & $3.5 \times 10^{-11}$ & $\mathrm{~b}$ \\
\hline $\mathrm{HEX} 2 \mathrm{ONE}+\mathrm{Cl} \rightarrow \mathrm{HEX} 2 \mathrm{ONCO} 2+\mathrm{HCl}$ & $2.7 \times 10^{-11}$ & $\mathrm{~b}$ \\
\hline $\mathrm{HEX} 3 \mathrm{ONE}+\mathrm{Cl} \rightarrow \mathrm{HEX} 3 \mathrm{ONAO} 2+\mathrm{HCl}$ & $1.05 \times 10^{-10}$ & $\mathrm{~b}$ \\
\hline $\mathrm{HEX} 3 \mathrm{ONE}+\mathrm{Cl} \rightarrow \mathrm{HEX} 3 \mathrm{ONBO} 2+\mathrm{HCl}$ & $2.3 \times 10^{-11}$ & $\mathrm{~b}$ \\
\hline $\mathrm{HEX} 3 \mathrm{ONE}+\mathrm{Cl} \rightarrow \mathrm{HEX} 3 \mathrm{ONCO} 2+\mathrm{HCl}$ & $1.8 \times 10^{-11}$ & $\mathrm{~b}$ \\
\hline $\mathrm{HEX} 3 \mathrm{ONE}+\mathrm{Cl} \rightarrow \mathrm{HEX} 3 \mathrm{ONDO} 2+\mathrm{HCl}$ & $1.8 \times 10^{-11}$ & $\mathrm{~b}$ \\
\hline $\mathrm{MIBK}+\mathrm{Cl} \rightarrow \mathrm{MIBKAO} 2+\mathrm{HCl}$ & $3.1 \times 10^{-10}$ & $\mathrm{~b}$ \\
\hline $\mathrm{MIBK}+\mathrm{Cl} \rightarrow \mathrm{MIBKBO} 2+\mathrm{HCl}$ & $3.0 \times 10^{-11}$ & $\mathrm{~b}$ \\
\hline $\mathrm{MTBK}+\mathrm{Cl} \rightarrow \mathrm{MTBKO} 2+\mathrm{HCl}$ & $2.9 \times 10^{-11}$ & $\mathrm{~b}$ \\
\hline
\end{tabular}


Alcohols $+\mathrm{Cl}$

$\mathrm{CH} 3 \mathrm{OH}+\mathrm{Cl} \rightarrow \mathrm{HO} 2+\mathrm{HCHO}+\mathrm{HCl}$

$\mathrm{C} 2 \mathrm{H} 5 \mathrm{OH}+\mathrm{Cl} \rightarrow \mathrm{CH} 3 \mathrm{CHO}+\mathrm{HO} 2+\mathrm{HCl}$

$\mathrm{C} 2 \mathrm{H} 5 \mathrm{OH}+\mathrm{Cl} \rightarrow \mathrm{HOCH} 2 \mathrm{CH} 2 \mathrm{O} 2+\mathrm{HCl}$

$\mathrm{NPROPOL}+\mathrm{Cl} \rightarrow \mathrm{C} 2 \mathrm{H} 5 \mathrm{CHO}+\mathrm{HO} 2+\mathrm{HCl}$

$\mathrm{NPROPOL}+\mathrm{Cl} \rightarrow \mathrm{HO} 1 \mathrm{C} 3 \mathrm{O} 2+\mathrm{HCl}$

$\mathrm{NPROPOL}+\mathrm{Cl} \rightarrow \mathrm{HYPROPO} 2+\mathrm{HCl}$

$\mathrm{IPROPOL}+\mathrm{Cl} \rightarrow \mathrm{CH} 3 \mathrm{COCH} 3+\mathrm{HO} 2+$

$\mathrm{HCl}$

$\mathrm{IPROPOL}+\mathrm{Cl} \rightarrow$ IPROPOLO $2+\mathrm{HCl}$

$\mathrm{NBUTOL}+\mathrm{Cl} \rightarrow \mathrm{C} 3 \mathrm{H} 7 \mathrm{CHO}+\mathrm{HO} 2+\mathrm{HCl}$

$\mathrm{NBUTOL}+\mathrm{Cl} \rightarrow \mathrm{NBUTOLAO} 2+\mathrm{HCl}$

$\mathrm{NBUTOL}+\mathrm{Cl} \rightarrow \mathrm{NBUTOLBO} 2+\mathrm{HCl}$

$\mathrm{BUT} 2 \mathrm{OL}+\mathrm{Cl} \rightarrow \mathrm{BUT} 2 \mathrm{OLO} 2+\mathrm{HCl}$

$\mathrm{BUT} 2 \mathrm{OL}+\mathrm{Cl} \rightarrow \mathrm{MEK}+\mathrm{HO} 2+\mathrm{HCl}$

$\mathrm{IBUTOL}+\mathrm{Cl} \rightarrow \mathrm{IBUTOLBO} 2+\mathrm{HCl}$

$\mathrm{IBUTOL}+\mathrm{Cl} \rightarrow \mathrm{IBUTOLCO} 2+\mathrm{HCl}$

$\mathrm{IBUTOL}+\mathrm{Cl} \rightarrow \mathrm{IPRCHO}+\mathrm{HO} 2+\mathrm{HCl}$

TBUTOL $+\mathrm{Cl} \rightarrow$ TBUTOLO $2+\mathrm{HCl}$

TBUTOL $+\mathrm{Cl} \rightarrow$ TC4H9O $+\mathrm{HCl}$

$\mathrm{PECOH}+\mathrm{Cl} \rightarrow \mathrm{DIEK}+\mathrm{HO} 2+\mathrm{HCl}$

$\mathrm{PECOH}+\mathrm{Cl} \rightarrow \mathrm{HO} 3 \mathrm{C} 5 \mathrm{O} 2+\mathrm{HCl}$

$\mathrm{PECOH}+\mathrm{Cl} \rightarrow \mathrm{PE} 2 \mathrm{ENEBO} 2+\mathrm{HCl}$

$\mathrm{IPEAOH}+\mathrm{Cl} \rightarrow \mathrm{BUT} 2 \mathrm{CHO}+\mathrm{HO} 2+\mathrm{HCl}$

$\mathrm{IPEAOH}+\mathrm{Cl} \rightarrow \mathrm{HM} 2 \mathrm{C} 43 \mathrm{O} 2+\mathrm{HCl}$

$\mathrm{IPEAOH}+\mathrm{Cl} \rightarrow \mathrm{M} 2 \mathrm{BUOL} 2 \mathrm{O} 2+\mathrm{HCl}$

$\mathrm{ME} 3 \mathrm{BUOL}+\mathrm{Cl} \rightarrow \mathrm{C} 3 \mathrm{ME} 3 \mathrm{CHO}+\mathrm{HO} 2+\mathrm{HCl}$

$\mathrm{ME} 3 \mathrm{BUOL}+\mathrm{Cl} \rightarrow \mathrm{HM} 33 \mathrm{C} 3 \mathrm{O} 2+\mathrm{HCl}$

$\mathrm{ME} 3 \mathrm{BUOL}+\mathrm{Cl} \rightarrow \mathrm{ME} 3 \mathrm{BUOLO} 2+\mathrm{HCl}$

$\mathrm{IPECOH}+\mathrm{Cl} \rightarrow \mathrm{HO} 2 \mathrm{M} 2 \mathrm{C} 4 \mathrm{O} 2+\mathrm{HCl}$

$\mathrm{IPECOH}+\mathrm{Cl} \rightarrow \mathrm{ME} 2 \mathrm{BU} 2 \mathrm{OLO} 2+\mathrm{HCl}$

$\mathrm{IPECOH}+\mathrm{Cl} \rightarrow \mathrm{PROL} 11 \mathrm{MO} 2+\mathrm{HCl}$

$\mathrm{IPEBOH}+\mathrm{Cl} \rightarrow \mathrm{H} 2 \mathrm{M} 3 \mathrm{C} 4 \mathrm{O} 2+\mathrm{HCl}$

$\mathrm{IPEBOH}+\mathrm{Cl} \rightarrow \mathrm{ME} 2 \mathrm{BUOLO} 2+\mathrm{HCl}$

$\mathrm{IPEBOH}+\mathrm{Cl} \rightarrow \mathrm{MIPK}+\mathrm{HO} 2+\mathrm{HCl}$

$\mathrm{CYHEXOL}+\mathrm{Cl} \rightarrow \mathrm{CYHEXOLAO} 2+\mathrm{HCl}$

$\mathrm{CYHEXOL}+\mathrm{Cl} \rightarrow \mathrm{CYHEXONE}+\mathrm{HO} 2+\mathrm{HCl}$
$7.1 \times 10^{-11} \times \exp (-75 / \mathrm{T}) \quad$ IUPAC

$5.5 \times 10^{-11} \times \exp (155 / \mathrm{T}) \quad$ IUPAC

$4.8 \times 10^{-12} \times \exp (155 / \mathrm{T}) \quad$ IUPAC

$1.6 \times 10^{-11} \times \exp (525 / \mathrm{T}) \quad$ IUPAC

$4.1 \times 10^{-12} \times \exp (525 / \mathrm{T}) \quad$ IUPAC

$6.8 \times 10^{-12} \times \exp (525 / \mathrm{T}) \quad$ IUPAC

$7.4 \times 10^{-11} \quad$ IUPAC

$1.3 \times 10^{-11} \quad$ IUPAC

$1.25 \times 10^{-11} \times \exp (550 / \mathrm{T}) \quad$ IUPAC

$1.12 \times 10^{-11} \times \exp (550 / \mathrm{T}) \quad$ IUPAC

$1.12 \times 10^{-11} \times \exp (550 / \mathrm{T}) \quad$ IUPAC

$5.37 \times 10^{-11} \quad \mathrm{~b}$

$9.51 \times 10^{-11} \quad b$

$2.6 \times 10^{-11} \times \exp (352 / \mathrm{T}) \quad \mathrm{b}$

$4.2 \times 10^{-12} \times \exp (352 / \mathrm{T}) \quad$ b

$1.6 \times 10^{-11} \times \exp (352 / \mathrm{T}) \quad \mathrm{b}$

$2.4 \times 10^{-11} \times \exp (-121 / \mathrm{T}) \quad \mathrm{b}$

$3.1 \times 10^{-12} \times \exp (-121 / \mathrm{T})$

$9.1 \times 10^{-11} \quad b$

$1.5 \times 10^{-11} \quad \mathrm{~b}$

$1.0 \times 10^{-10} \quad \mathrm{~b}$

$5.5 \times 10^{-11} \quad \mathrm{~b}$

$4.9 \times 10^{-11} \quad \mathrm{~b}$

$8.7 \times 10^{-11} \quad \mathrm{~b}$

$6.45 \times 10^{-11} \quad \mathrm{~b}$

$1.02 \times 10^{-10} \quad \mathrm{~b}$

$5.78 \times 10^{-11} \quad \mathrm{~b}$

$6.58 \times 10^{-12} \quad b$

$4.62 \times 10^{-11} \quad \mathrm{~b}$

$1.31 \times 10^{-11} \quad \mathrm{~b}$

$1.57 \times 10^{-11} \quad b$

$9.82 \times 10^{-11} \quad \mathrm{~b}$

$9.82 \times 10^{-11} \quad$ b

$2.2 \times 10^{-10} \quad b$

$7.9 \times 10^{-11} \quad$ b 


\begin{tabular}{|c|c|c|}
\hline $\mathrm{MIBKAOH}+\mathrm{Cl} \rightarrow$ MIBKAOHAO2+ $\mathrm{HCl}$ & $3.4 \times 10^{-11}$ & $\mathrm{~b}$ \\
\hline $\mathrm{MIBKAOH}+\mathrm{Cl} \rightarrow \mathrm{MIBKAOHBO} 2+\mathrm{HCl}$ & $1.3 \times 10^{-11}$ & $\mathrm{~b}$ \\
\hline $\mathrm{MIBKAOH}+\mathrm{Cl} \rightarrow \mathrm{MIBKHO} 4 \mathrm{O} 2+\mathrm{HCl}$ & $1.8 \times 10^{-12}$ & $\mathrm{~b}$ \\
\hline $\mathrm{ETHGLY}+\mathrm{Cl} \rightarrow \mathrm{HOCH} 2 \mathrm{CHO}+\mathrm{HO} 2+\mathrm{HCl}$ & $2.5 \times 10^{-10}$ & $\mathrm{~b}$ \\
\hline $\mathrm{PROPGLY}+\mathrm{Cl} \rightarrow \mathrm{ACETOL}+\mathrm{HO} 2+\mathrm{HCl}$ & $1.26 \times 10^{-10}$ & $\mathrm{~b}$ \\
\hline $\begin{array}{l}\mathrm{PROPGLY}+\mathrm{Cl} \rightarrow \mathrm{CH} 3 \mathrm{CHOHCHO}+\mathrm{HO} 2+ \\
\mathrm{HCl}\end{array}$ & $7.94 \times 10^{-11}$ & $\mathrm{~b}$ \\
\hline \multicolumn{3}{|c|}{ Organic acids $+\mathrm{Cl}$} \\
\hline $\mathrm{CH} 3 \mathrm{OOH}+\mathrm{Cl} \rightarrow \mathrm{CH} 3 \mathrm{O} 2+\mathrm{HCl}$ & $3.54 \times 10^{-11}$ & IUPAC \\
\hline $\mathrm{CH} 3 \mathrm{OOH}+\mathrm{Cl} \rightarrow \mathrm{HCHO}+\mathrm{OH}+\mathrm{HCl}$ & $2.36 \times 10^{-11}$ & IUPAC \\
\hline $\mathrm{HCOOH}+\mathrm{Cl} \rightarrow \mathrm{HO} 2+\mathrm{HCl}$ & $1.9 \times 10^{-13}$ & IUPAC \\
\hline $\mathrm{CH} 3 \mathrm{CO} 2 \mathrm{H}+\mathrm{Cl} \rightarrow \mathrm{CH} 3 \mathrm{O} 2+\mathrm{HCl}$ & $2.65 \times 10^{-14}$ & IUPAC \\
\hline $\mathrm{PROPACID}+\mathrm{Cl} \rightarrow \mathrm{C} 2 \mathrm{H} 5 \mathrm{O} 2+\mathrm{HCl}$ & $3.96 \times 10^{-14}$ & $\mathrm{~b}$ \\
\hline \multicolumn{3}{|c|}{ Organic nitrates $+\mathrm{Cl}$} \\
\hline $\mathrm{CH} 3 \mathrm{NO} 3+\mathrm{Cl} \rightarrow \mathrm{HCHO}+\mathrm{NO} 2+\mathrm{HCl}$ & $2.4 \times 10^{-13}$ & IUPAC \\
\hline $\mathrm{C} 2 \mathrm{H} 5 \mathrm{NO} 3+\mathrm{Cl} \rightarrow \mathrm{CH} 3 \mathrm{CHO}+\mathrm{NO} 2+\mathrm{HCl}$ & $4.7 \times 10^{-12}$ & IUPAC \\
\hline $\begin{array}{l}\mathrm{NC} 3 \mathrm{H} 7 \mathrm{NO} 3+\mathrm{Cl} \rightarrow \mathrm{C} 2 \mathrm{H} 5 \mathrm{CHO}+\mathrm{NO} 2+ \\
\mathrm{HCl}\end{array}$ & $2.2 \times 10^{-11}$ & IUPAC \\
\hline $\begin{array}{l}\mathrm{IC} 3 \mathrm{H} 7 \mathrm{NO} 3+\mathrm{Cl} \rightarrow \mathrm{CH} 3 \mathrm{COCH} 3+\mathrm{NO} 2+ \\
\mathrm{HCl}\end{array}$ & $3.8 \times 10^{-12}$ & IUPAC \\
\hline $\begin{array}{l}\mathrm{NC} 4 \mathrm{H} 9 \mathrm{NO} 3+\mathrm{Cl} \rightarrow \mathrm{C} 3 \mathrm{H} 7 \mathrm{CHO}+\mathrm{NO} 2+ \\
\mathrm{HCl}\end{array}$ & $8.5 \times 10^{-11}$ & IUPAC \\
\hline
\end{tabular}

\section{Aromatics $+C l$}

$$
\begin{aligned}
& \text { TOLUENE }+\mathrm{Cl} \rightarrow \mathrm{C} 6 \mathrm{H} 5 \mathrm{CH} 2 \mathrm{O} 2+\mathrm{HCl} \\
& \mathrm{OXYL}+\mathrm{Cl} \rightarrow \mathrm{OXYLO} 2+\mathrm{HCl} \\
& \mathrm{MXYL}+\mathrm{Cl} \rightarrow \mathrm{MXYLO} 2+\mathrm{HCl} \\
& \mathrm{PXYL}+\mathrm{Cl} \rightarrow \mathrm{PXYLO} 2+\mathrm{HCl} \\
& \mathrm{EBENZ}+\mathrm{Cl} \rightarrow \mathrm{C} 6 \mathrm{H} 5 \mathrm{C} 2 \mathrm{H} 4 \mathrm{O} 2+\mathrm{HCl} \\
& \mathrm{PBENZ}+\mathrm{Cl} \rightarrow \mathrm{PHC} 3 \mathrm{O} 2+\mathrm{HCl} \\
& \mathrm{IPBENZ}+\mathrm{Cl} \rightarrow \mathrm{PHIC} 3 \mathrm{O} 2+\mathrm{HCl} \\
& \text { TM123B }+\mathrm{Cl} \rightarrow \mathrm{TM} 123 \mathrm{BO} 2+\mathrm{HCl} \\
& \text { TM124B }+\mathrm{Cl} \rightarrow \mathrm{TM} 124 \mathrm{BO} 2+\mathrm{HCl} \\
& \mathrm{TM} 135 \mathrm{~B}+\mathrm{Cl} \rightarrow \mathrm{TMBO} 2+\mathrm{HCl} \\
& \mathrm{OETHTOL}+\mathrm{Cl} \rightarrow \mathrm{ETOLO} 2+\mathrm{HCl} \\
& \text { METHTOL }+\mathrm{Cl} \rightarrow \mathrm{ETOLO} 2+\mathrm{HCl} \\
& \text { PETHTOL }+\mathrm{Cl} \rightarrow \text { ETOLO} 2+\mathrm{HCl}
\end{aligned}
$$$$
5.9 \times 10^{-11}
$$$$
1.5 \times 10^{-10}
$$$$
1.7 \times 10^{-10}
$$$$
2.6 \times 10^{-10}
$$$$
9.1 \times 10^{-11}
$$$$
\text { Shi and }
$$$$
\text { Bernhard, } 1997
$$$$
\text { Shi and }
$$$$
\text { Bernhard, } 1997
$$$$
7.5 \times 10^{-11}
$$$$
8.2 \times 10^{-11}
$$$$
3.6 \times 10^{-10}
$$$$
3.6 \times 10^{-10}
$$$$
3.1 \times 10^{-10}
$$$$
1.1 \times 10^{-10}
$$$$
\text { b }
$$$$
\text { b }
$$$$
\text { b }
$$$$
\text { b }
$$$$
\text { b }
$$$$
\text { b }
$$$$
\text { b }
$$$$
\text { b }
$$$$
\text { b }
$$$$
1.4 \times 10^{-10} \quad \mathrm{~b}
$$$$
2.2 \times 10^{-10}
$$

\section{b} b b b b ( (1) b b b b 
Alkenes $+\mathrm{Cl}^{\mathrm{C}}$

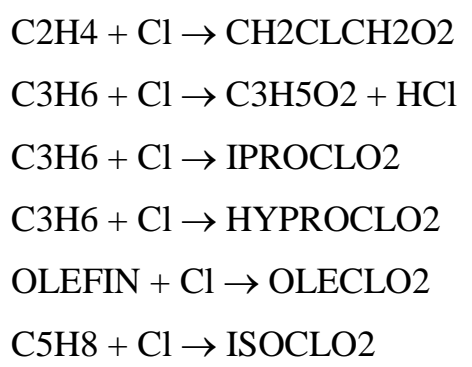

\begin{tabular}{cc}
$1.0 \times 10^{-10}$ & IUPAC \\
$2.7 \times 10^{-11}$ & Riedel et al. 2014 \\
$1.35 \times 10^{-10}$ & Riedel et al. 2014 \\
$1.08 \times 10^{-10}$ & Riedel et al. 2014 \\
$1.16 \times 10^{-9}$ & CB-IV \\
$1.28 \times 10^{-10} \times \exp (390 / \mathrm{T})$ & CB-IV \\
\hline
\end{tabular}

a All species are variables existing in the MCM, except for "OLEFIN" and the reaction products of $\mathrm{Cl}$ with $\mathrm{C}_{3} \mathrm{H}_{6}$, OLEFIN and $\mathrm{C}_{5} \mathrm{H}_{8}$.

${ }^{\mathrm{b}}$ Reaction with $\mathrm{Cl}$ is assumed to be similar to that with $\mathrm{OH}$, and the $\mathrm{Cl}$ rate constant is calculated by multiplying the $\mathrm{OH}$ rate constant by the average $k_{C l} / k_{O H}$ for compounds with available kinetic data (note that the $\mathrm{C}_{1}$ species was excluded from the $k_{C l} / k_{O H}$ calculation).

${ }^{\mathrm{C}}$ Reactions of $\mathrm{Cl}$ with $\mathrm{C}_{3} \mathrm{H}_{6}$, OLEFIN and $\mathrm{C}_{5} \mathrm{H}_{8}$ introduce new model species and further reactions, which are represented in detail in Figures 1-3 in the main text.

IUPAC: International Union of Pure and Applied Chemistry (http://iupac.pole-ether.fr/index.html); CB-IV: Carbon Bond Mechanism IV (Tanaka et al., 2003). 
Table S2. Initial model conditions for simulations of the urban plum observed at Hok Tsui, Hong Kong during 24 August 2012.

\begin{tabular}{|c|c|c|c|}
\hline Species & Concentration ${ }^{a}$ & Species & Concentration $^{a}$ \\
\hline Temperature $^{\mathrm{b}}$ & $301 \mathrm{~K}$ & $\mathrm{C}_{3} \mathrm{H}_{6}$ & 280 \\
\hline Pressure & $760 \mathrm{~mm} \mathrm{Hg}$ & 1-butene & 80 \\
\hline $\mathrm{H}_{2} \mathrm{O}^{\mathrm{b}}$ & $0.028 \% / \%$ & cis-2-butene & 4 \\
\hline NO & 20 & trans-2-butene & 40 \\
\hline $\mathrm{NO}_{2}$ & $15.6 \mathrm{ppb}$ & trans-2-pentene & 45 \\
\hline $\mathrm{O}_{3}$ & $64 \mathrm{ppb}$ & 1,3-butadiene & 40 \\
\hline PAN & 2100 & 1-pentene & 45 \\
\hline $\mathrm{SO}_{2}$ & $5.0 \mathrm{ppb}$ & Isoprene & 4 \\
\hline HONO & 814 & $\mathrm{C}_{2} \mathrm{H}_{2}$ & 2150 \\
\hline $\mathrm{ClNO}_{2}$ & 1997 & Benzene & 390 \\
\hline $\mathrm{CO}$ & $297 \mathrm{ppb}$ & Toluene & 1130 \\
\hline $\mathrm{CH}_{4}$ & $2.01 \mathrm{ppm}$ & $o$-xylene & 110 \\
\hline $\mathrm{C}_{2} \mathrm{H}_{6}$ & 1860 & $m, p$-xylene & 260 \\
\hline $\mathrm{C}_{3} \mathrm{H}_{8}$ & 2840 & Ethylbenzene & 280 \\
\hline$n$-butane & 2640 & 1,2,3-trimethylbenzene & 6 \\
\hline$i$-butane & 1640 & 1,2,4-trimethylbenzene & 6 \\
\hline$n$-pentane & 440 & 1,3,5-trimethylbenzene & 5 \\
\hline$i$-pentane & 710 & $\mathrm{HCHO}$ & 2790 \\
\hline$n$-hexane & 71 & $\mathrm{CH}_{3} \mathrm{CHO}$ & 1180 \\
\hline 2-methylpentane & 18 & $\mathrm{C}_{2} \mathrm{H}_{5} \mathrm{CHO}$ & 114 \\
\hline$n$-heptane & 61 & Benzaldehyde & 120 \\
\hline$n$-octane & 470 & acetone & 1100 \\
\hline 2,2,4-trimethylpentane & 610 & MEK & 137 \\
\hline $\mathrm{C}_{2} \mathrm{H}_{4}$ & 560 & & \\
\hline
\end{tabular}

${ }^{\mathrm{a}}$ The unit is pptv unless otherwise specified.

${ }^{\mathrm{b}}$ For temperature, water content and $\mathrm{J}_{\mathrm{NO} 2}$, the measured diurnal profiles at Hok Tsui were used to constrain the model. 


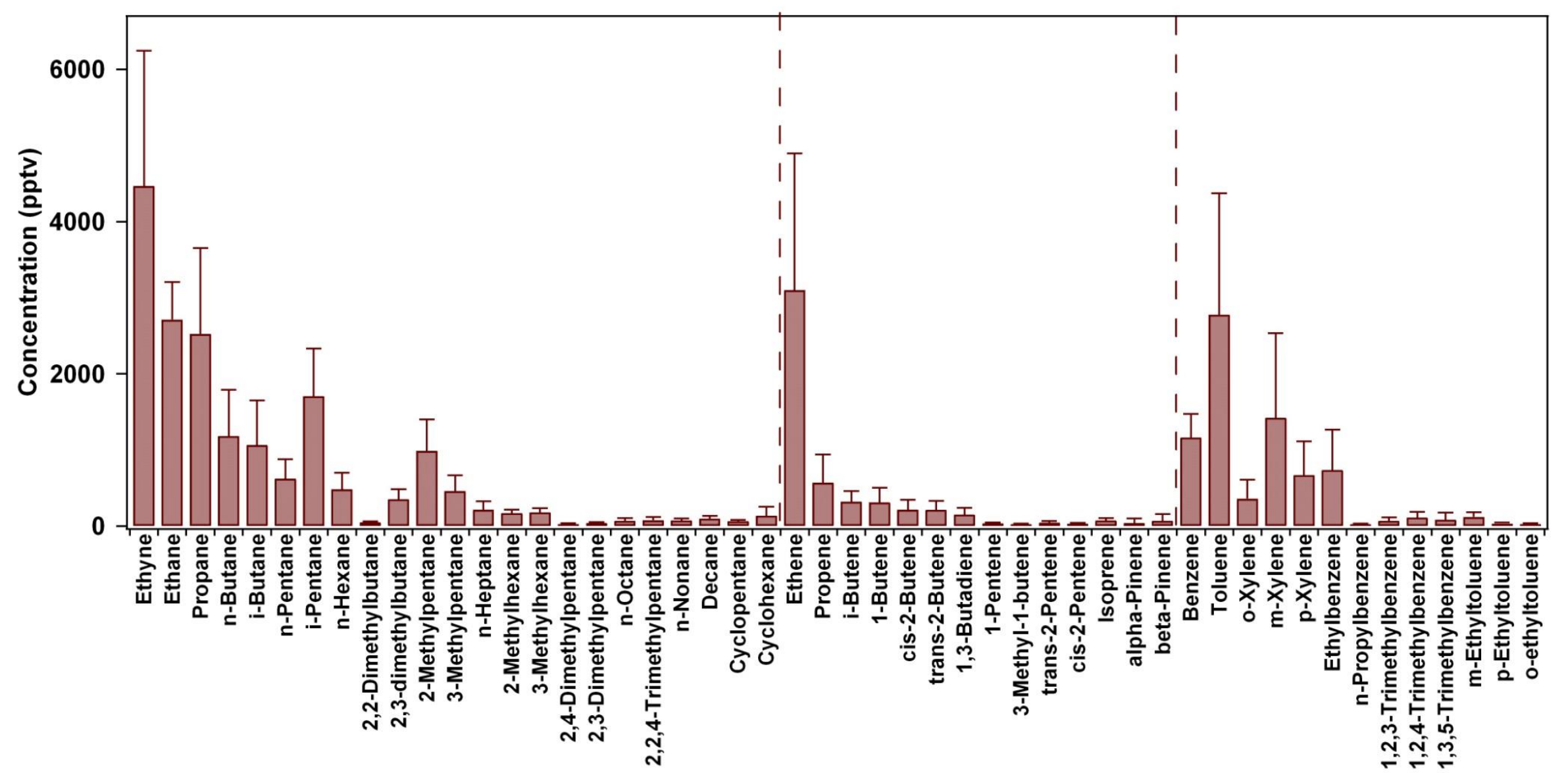

Figure S1. Average concentrations of individual hydrocarbon species observed at Shanghai on 7 May 2005 when the data were used for sensitivity tests of the new $\mathrm{Cl} \cdot$ mechanism. Error bars refer to standard deviations. 


\section{Description of the high $\mathrm{CINO}_{2}$ plume and intensive observations at Hok Tsui}

Field observations were conducted from 23 August to 1 September 2012 at Hok Tsui $\left(22^{\circ} 13^{\prime} \mathrm{N}, 114^{\circ} 15^{\prime} \mathrm{E}, 60 \mathrm{~m}\right.$ above sea level), a coastal site at the southeast tip of Hong Kong Island. This site receives either polluted plumes from urban Hong Kong and/or the Pearl River Delta region under northerly winds or clean marine air masses when southerly winds dominate. It has been deployed as the sampling platform in many previous studies (e.g., Wang et al., 2009; Zha et al., 2014). A full list of chemicals and meteorological parameters were measured in-situ during this intensive study.

$\mathrm{ClNO}_{2}$ and $\mathrm{N}_{2} \mathrm{O}_{5}$ were concurrently measured by an iodide-chemical ionization mass spectrometer (CIMS) (THS Instruments Inc., Atlanta), in which the target molecules are ionized to iodide clusters followed by detection by a quadruple mass spectrometer. The detailed descriptions of the instrument principles and operation/calibration procedures have been provided elsewhere (Tham et al., 2014; Wang et al., 2015). Ozone was measured by a commercial UV photometric analyzer (TEI, Model 49i). Carbon monoxide was measured with a non-dispersive infrared analyzer (API, Model 300EU). Nitrogen oxides ( $\mathrm{NO}$ and $\mathrm{NO}_{2}$ ) were measured with a chemiluminescence instrument (TEI, Model 42i) equipped with a photolytic converter. NOy was determined by another chemiluminescence analyzer (TEI, Model $42 i$ ) with an externally placed molybdenum oxide (MoO) catalytic converter. $\mathrm{C}_{2}-\mathrm{C}_{10}$ hydrocarbons were measured in real-time by a commercial analyzer that combines gas chromatography (GC) separation, photoionization detection (PID), and flame-ionization detection (FID) (Syntech Spectras, model GC955 Series 600/800 POCP). In addition, whole air samples were also collected on selected days for detection of $\mathrm{C}_{1}-\mathrm{C}_{10}$ hydrocarbons, and analyzed at the University of California, Irvine (UCI) laboratory (Xue et al., 2013). $\mathrm{C}_{1}-\mathrm{C}_{8}$ carbonyls were measured by collecting air samples in sorbent cartridges coated by 2,4-dinitrophenylhydrazine (DNPH) equipped with an $\mathrm{O}_{3}$ scrubber, followed by analysis by a high pressure liquid chromatography (Xue et al., 2014). HONO was measured in real-time using a long path absorption photometer (LOPAP). Temperature, relative humidity, wind speed and direction, and $\mathrm{J}_{\mathrm{NO} 2}$ values were also measured by commercial probes/sensors (Zha et al., 2014).

The time series of $\mathrm{ClNO}_{2}, \mathrm{O}_{3}, \mathrm{NO}_{2}$ and $J_{\mathrm{NO} 2}$ is depicted in Figure $S 2$. The plume with the highest $\mathrm{ClNO}_{2}$ pollution was observed during the night of 23-24 August. The maximum value of $\mathrm{ClNO}_{2}$ was recorded as 1997 pptv (1-min data) at around 3:00 AM local time on 24 August. Such a level is among the highest values ever reported over the world. Examination of surface winds and backward trajectories suggested that this polluted plume originated from urban Hong Kong and the Pearl River Delta region. This case has been analyzed in detail in our previous work (Tham et al., 2014). 


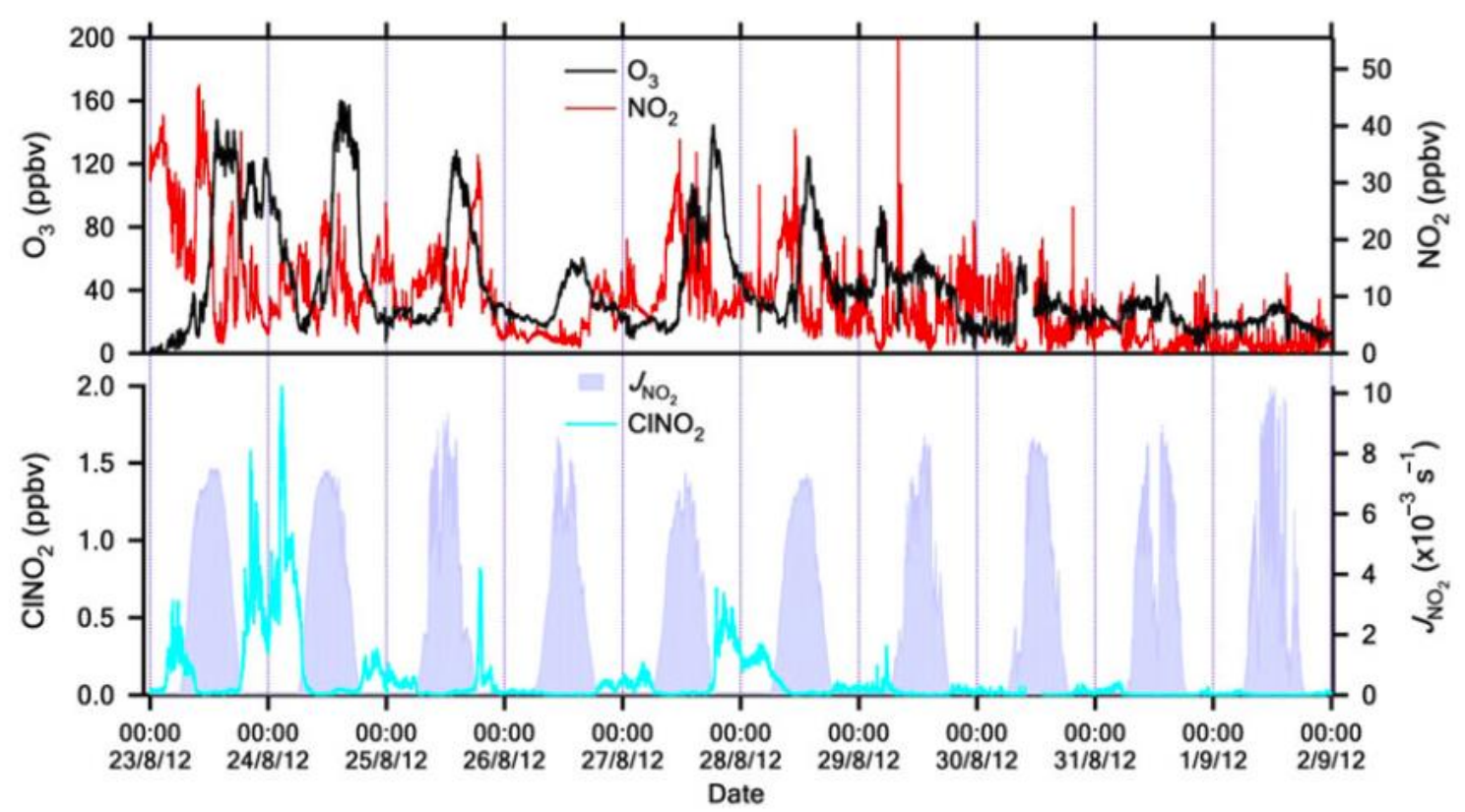

Figure S2. Time series of $\mathrm{ClNO}_{2}, \mathrm{O}_{3}, \mathrm{NO}_{2}$ and $\mathrm{J}_{\mathrm{NO} 2}$ observed at Hok Tsui in summer 2012. (from Tham et al. 2014).

\section{References}

Riedel, T. P., Wolfe, G. M., Danas, K. T., Gilman, J. B., Kuster, W. C., Bon, D. M., Vlasenko, A., Li, S. M., Williams, E. J., Lerner, B. M., Veres, P. R., Roberts, J. M., Holloway, J. S., Lefer, B., Brown, S. S., and Thornton, J. A.: An MCM modeling study of nitryl chloride $\left(\mathrm{ClNO}_{2}\right)$ impacts on oxidation, ozone production and nitrogen oxide partitioning in polluted continental outflow, Atmos Chem Phys, 14, 3789-3800, 2014.

Shi, J. C. and Bernhard, M. J.: Kinetic studies of Cl-atom reactions with selected aromatic compounds using the photochemical reactor-FTIR spectroscopy technique, Int J Chem Kinet, 29, 349-358, 1997.

Tanaka, P. L., Allen, D. T., McDonald-Buller, E. C., Chang, S. H., Kimura, Y., Mullins, C. B., Yarwood, G., and Neece, J. D.: Development of a chlorine mechanism for use in the carbon bond IV chemistry model, J Geophys Res-Atmos, 108, 2003.

Tham, Y. J., Yan, C., Xue, L. K., Zha, Q. Z., Wang, X. F., and Wang, T.: Presence of high nitryl chloride in Asian coastal environment and its impact on atmospheric photochemistry, Chinese Sci Bull, 59, 356-359, 2014.

Wang, T., Wei, X. L., Ding, A. J., Poon, C. N., Lam, K. S., Li, Y. S., Chan, L. Y., and Anson, M.: Increasing surface ozone concentrations in the background atmosphere of Southern China, 1994-2007, Atmos. Chem. Phys., 9, 6217-6227, 2009.

Wang, T., Tham, Y. J., Xue, L. K., Li, Q. Y., Zha, Q. Z., Wang, Z., Poon, S. C. N., Dube, W. P., 
Brown, S. S., Louie, P. K. K., Luk, C. W. Y., Blake, D. R., Tsui, W.: High levels of nitryl chloride in the planetary boundary layer: A case study at a mountain site in Hong Kong, Geophys. Res. Lett., submitted, 2015.

Xue, L. K., Wang, T., Guo, H., Blake, D. R., Tang, J., Zhang, X. C., Saunders, S. M., and Wang, W. X.: Sources and photochemistry of volatile organic compounds in the remote atmosphere of western China: results from the Mt. Waliguan Observatory, Atmos Chem Phys, 13, 8551-8567, 2013.

Xue, L. K., Wang, T., Wang, X. F., Blake, D. R., Gao, J., Nie, W., Gao, R., Gao, X. M., Xu, Z., Ding, A. J., Huang, Y., Lee, S. C., Chen, Y. Z., Wang, S. L., Chai, F. H., Zhang, Q. Z., and Wang, W. X. On the use of an explicit chemical mechanism to dissect peroxy acetyl nitrate formation, Environmental Pollution, 195, 39-47, 2014.

Zha, Q. Z., Xue, L. K., Wang, T., et al., Large conversion rates of $\mathrm{NO}_{2}$ to $\mathrm{HNO}_{2}$ observed in air masses from the South China Sea: evidence of strong production at sea surface?, Geophys. Res. Lett., 41, 7710- 7715, 2014. 